\title{
Sclerosing mediastinitis: a report on 18 cases
}

\author{
T M Mole, J Glover, M N Sheppard
}

\begin{abstract}
Background - Sclerosing mediastinitis is a rare condition which causes dense fibrosis of the mediastinum. Few large studies have been reported to date. The clinical and pathological features of cases have been studied in a specialist referral centre in the UK.
\end{abstract}

Methods - The pathological files of the Royal Brompton Hospital were examined and 18 cases of sclerosing mediastinitis were identified between 1970 and 1993. The clinical notes were obtained and the pathological specimens analysed.

Results - There were 12 men and six women of age range 9-64 years. Twelve patients presented with shortness of breath, six had haemoptysis, three had hoarseness, four had pleuritic chest pain, three general weakness, two had dysphagia, and one was asymptomatic. Nine patients had a previous history of pulmonary tuberculosis. Two had autoimmune disease - one rheumatoid arthritis and the other systemic lupus erythematosus. There were three cases of previous malignancy - two undifferentiated carcinoma of the lung and the other Hodgkin's disease. Serological tests revealed only one positive reaction to Histoplasma. The erythrocyte sedimentation rate and serum immunoglobulins were raised in nine patients. Diagnosis was usually by thoracotomy with biopsy. All cases had fibrosis and chronic inflammation with no active granulomas. No infective organisms or positive cultures were obtained in any case. Ten of the 18 cases are alive up to 15 years after diagnosis, with only two deaths and six lost to follow up.

Conclusions - Sclerosing mediastinitis is a slowly progressive condition associated with previous tuberculosis, mediastinal malignancy, and autoimmune disease. The outlook is excellent for those cases without underlying malignancy.

(Thorax 1995;50:280-283)

Keywords: sclerosing mediastinitis, tuberculosis, histoplasmosis, autoimmune disease.

Sclerosing mediastinitis is a rare condition in which there is fibrosis and sclerosis of the mediastinum with resulting obstruction to intrathoracic organs. It was first reported by Oulmont in 1855 who classified it as idiopathic fibrous mediastinitis. ${ }^{1}$ In 1930 Osler described additional patients with mediastinal fibrosis of unclassified aetiology and superior vena caval obstruction. ${ }^{2}$ The condition was thought to be secondary to tuberculosis or syphilis until 1925 when Knox reported an association with fungal infections. ${ }^{3}$ It is very rare and there are few details concerning large groups of sclerosing mediastinitis. At the Royal Brompton Hospital we have collected, in the last 20 years, 18 cases of sclerosing mediastinitis. The object of this study was to detail the epidemiological, pathological, and clinical features of these cases.

Methods and results

The histopathology files from 1970 to 1993 were studied and 18 cases diagnosed as sclerosing mediastinitis were found. The case notes and all the histopathological sections of each case were studied. In each patient the age, sex, nationality, family history, drug exposure, previous disease, chemotherapy, radiotherapy, travel, radiology, investigations for infection, diagnosis, surgical procedure, and follow up were analysed.

\section{PATIENT DETAILS}

Twelve of the 18 patients were men; their age range was 9-64 years. Eleven $(62 \cdot 5 \%)$ were smokers. None had a family history of sclerosing mediastinitis but $12(66 \%)$ had a previous history of other disease. Two had well established autoimmune disease (one rheumatoid arthritis and the other systemic lupus erythematous), three had a previous malignancy (two non-small cell carcinoma of the lung with mediastinal lymph node involvement and one Hodgkin's disease), all diagnosed and treated at least three years before presentation of their mediastinal symptoms. These three patients had been given cytotoxic chemotherapy and radiotherapy to the chest. Nine patients had a history of previous pulmonary tuberculosis, one of whom also had rheumatoid arthritis, another non-small cell carcinoma of the lung, while one other had lived in the USA for five years in an area in which Histoplasma was endemic and seroconverted for Histoplasma whilst there after an influenza-like illness with respiratory symptoms. None had taken specific drugs associated with fibrogenesis, in particular methysergide.

The clinical features are summarised in the table. The highest documented erythrocyte sedimentation rate was $55 \mathrm{~mm} /$ hour. Details concerning BCG immunisation were not found in the case notes. Serological tests for hepatitis, fungi, mycoplasma, cytomegalovirus, influenza virus, adenovirus, and psittacosis were negative in all patients tested, except for the one case in whom the Histoplasma titre was positive. Radiological details are also given in the table. 
Clinical and radiological features of 18 patients with sclerosing mediastinitis

\begin{tabular}{lr}
\hline & $n$ \\
\hline Symptoms & \\
Shortness of breath & 12 \\
Haemoptysis & 6 \\
Dysphagia & 2 \\
Hoarseness & 3 \\
Pleuritic chest pain & 4 \\
Malaise & 3 \\
Asymptomatic & 1 \\
& \\
Investigations & 9 \\
ESR (16-55 mm/hour) & 9 \\
IgG (16-20 g/l) & +7 \\
Mantoux (17) & +1 \\
Histoplasma (6) & \\
Radiology & 9 \\
Mediastinal widening & 5 \\
Hilar widening & 7 \\
Calcification & 6 \\
Vena caval obstruction & \\
\hline
\end{tabular}

$\mathrm{ESR}=$ erythrocyte sedimentation rate.

Calcification of the mediastinal mass was noted in seven of the patients (fig 1). No evidence of active tuberculosis was seen. One case showed diffuse infiltration of the right middle lobe while another showed a large mass at the right hilum. Six patients had superior vena cavagrams, all of whom had clinical evidence of vena caval obstruction. The initial impression in all patients was of mediastinal malignancy.

\section{SURGICAL PROCEDURES}

Bronchoscopy was performed in all patients and in two cases there was evidence of external compression because the wall of the bronchus appeared to be distorted and narrowed but no endobronchial lesions were seen. Both provided sufficient biopsy material to make a diagnosis in keeping with sclerosing mediastinitis. Four patients proceeded to mediastinoscopy because of a mass at the hilum with probable paratracheal and subcarinal lymph node involvement. Eight patients had a median sternotomy, six for superior caval bypass grafting, one for routine coronary artery bypass grafting,

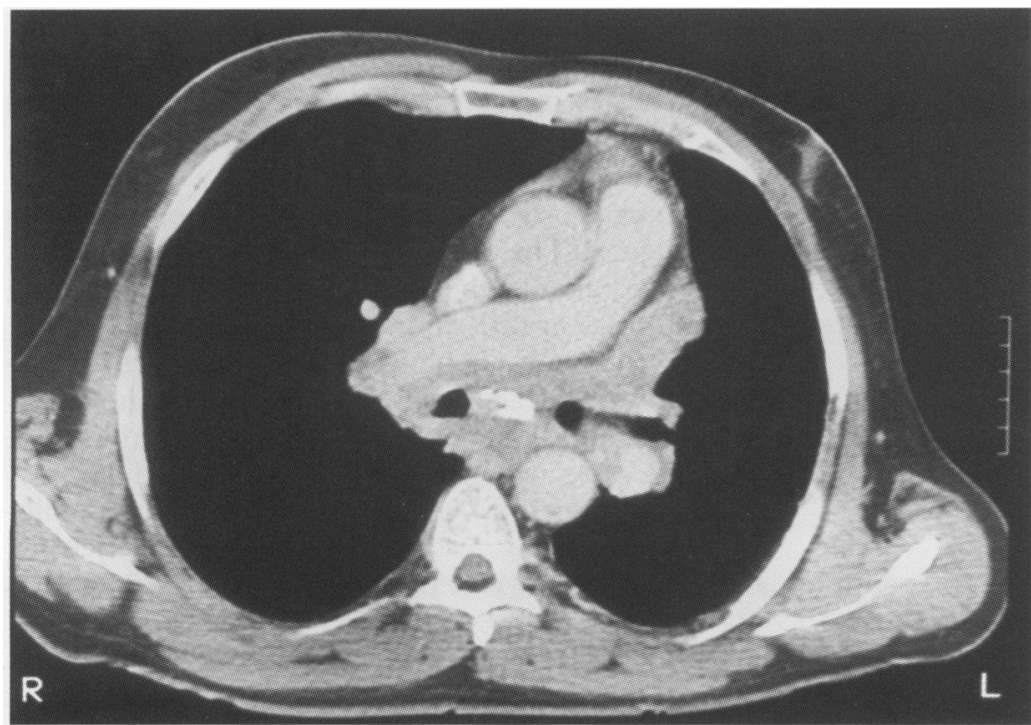

Figure 1 Computed tomographic scan through the mediastinum at the level of the aortic arch. A confluent abnormal soft tissue mass can be seen surrounding and deforming the main bronchi. A focus of calcification is seen in the subcarinal region, presumably within a lymph node mass. Previous history of tuberculosis.

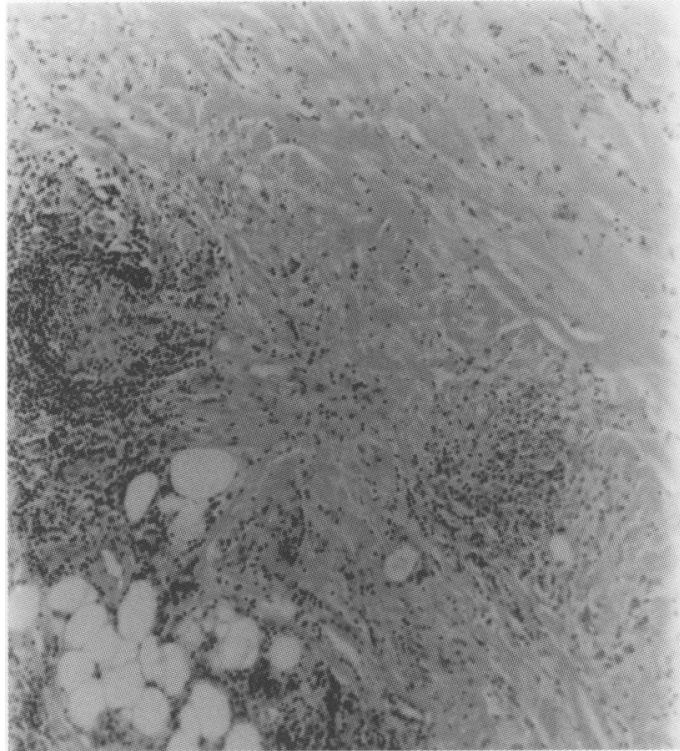

Figure 2 Section of tissue from mediastinum showing hyaline fibrosis in the centre with the periphery containing lymphoid follicles infiltrating fat. Original magnification $\times 200$ reduced to $61 \%$ in origination.

and another for a suspected thymic mass. Two patients had a thoracotomy, one for a right middle lobectomy and the other a right lower lobectomy for bronchiectasis associated with a right hilar mass. Two cases were diagnosed at necropsy. In each surgical case sufficient material was removed for diagnostic purposes but complete resection of the fibrous mass was impossible. In all cases who underwent surgery there was extensive involvement of thoracic organs by the fibrosing process, with the lungs being involved in 11 cases, the phrenic nerve in seven, the superior vena cava in eight, and the pulmonary artery in two. Six patients had a superior vena caval bypass with woven silicone prostheses (three cases), autogenous saphenous vein graft (two cases), and glutaraldehyde fixed bovine pericardial graft (one case) from the junction of the right and left brachiocephalic veins to the superior vena cava, bypassing the fibrous mediastinal mass. No stents were used in any of the grafts.

\section{PATHOLOGICAL FINDINGS}

One bronchial biopsy sample showed proliferation of dense fibrous tissue within the wall of the bronchus associated with plasma cells and lymphocytes, while a second sample showed chronic inflammmation only. These were considered sufficient evidence to make a probable diagnosis of sclerosing mediastinitis. During the other procedures several blocks of tissue taken from the mediastinum showed areas of dense hyaline sclerosis with interlacing bundles of fibrous tissue extending into loose connective tissue and fat (fig 2). The fibrous tissue often contained acellular hyaline fibrosis as its centre with an inflammatory edge including lymphocytes with lymphoid follicle formation. This fibrous proliferation extended into the lumen of the mediastinal blood vessels (fig 3). Admixed with the fibrous tissue were 


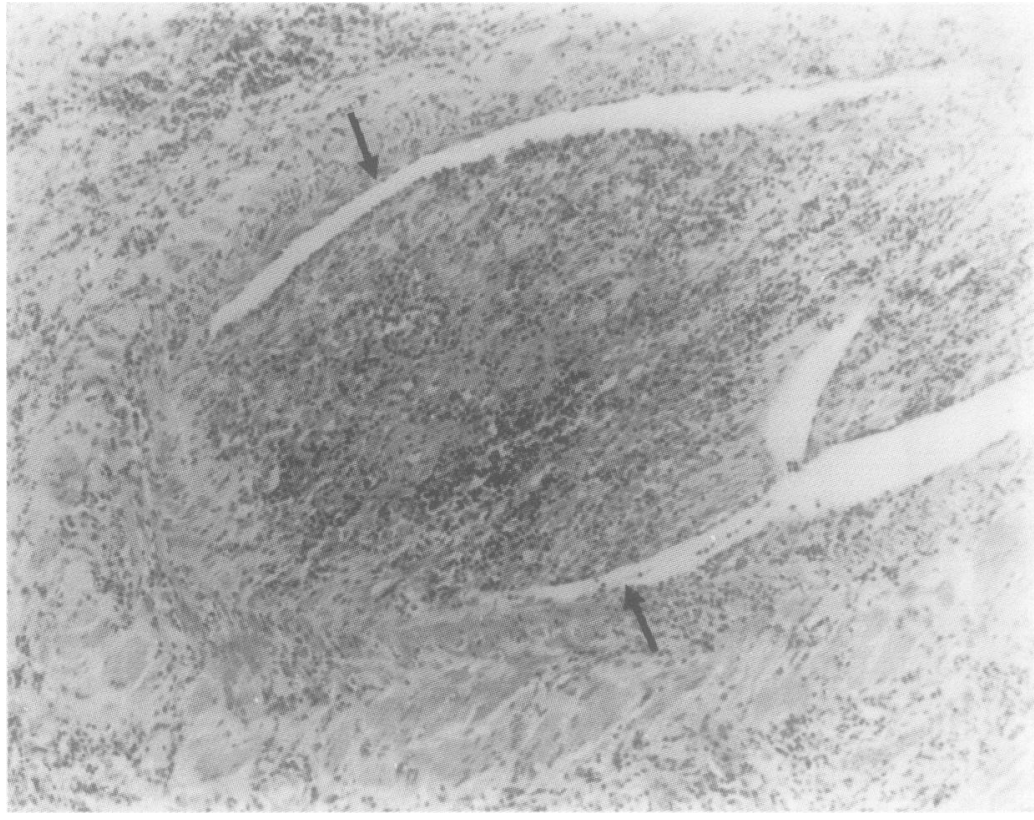

Figure 3 Inflammatory fibrous tissue containing plasma cells and lymphocytes infiltrating the wall and lumen (arrows) of a mediastinal vein. Original magnification $\times 250$, reduced to $71 \%$ in origination.

many plasma cells and lymphocytes. No necrosis, granulomas, or giant cells were noted in any of the sections examined. In the two pulmonary resection specimens the fibrosing process extended into the pleura and the interstitium of the lung. Appearances were identical to fibrosing alveolitis. Special stains for acid fast bacilli and fungi were negative in all tissues examined and cultures were also negative.

\section{FOLLOW ÜP}

Twelve patients were followed up for 5-20 years while six were lost to follow up. These six had been referred from abroad and contact was lost. Ten patients were alive and free from symptoms, including three who had superior vena caval bypass grafting. Death occurred in only two cases due to Hodgkin's disease and non-small cell bronchial carcinoma. The patient with Hodgkin's disease died of bronchopneumonia within two years of diagnosis; no residual tumour was found at necropsy but there was widespread fibrosis of the mediastinum with involvement of both lungs. The other patient also died of respiratory failure with an enlarged mediastinum but no necropsy was performed so the possibility of residual tumour could not be eliminated. There are no details available concerning the use of antituberculous and antifungal agents or steroids in any of the cases.

\section{Discussion}

Sclerosing mediastinitis is a rare condition and only 180 cases had been reported up to $1969 .{ }^{4}$ Since then reports in Europe have been of single cases or small groups as experience of this condition is limited. Among the accepted causes of sclerosing mediastinitis infection is the most common, especially fungal infections including histoplasmosis (which is the com- monest cause in the USA ${ }^{4}$ ), aspergillosis, mucormycosis, cryptococcosis, blastomycosis, and mycobacteria. Non-infectious causes ${ }^{5}$ include autoimmune disease, sarcoidosis, rheumatic fever, neoplasms such as Hodgkin's disease, traumatic haemorrhage and drugs, particularly methysergide. ${ }^{6}$ In rare instances it is familial and multifocal with patients presenting with retroperitoneal and mediastinal fibrosis, sclerosing cholangitis, Riedel's thyroiditis, and pseudotumour of the orbit. ${ }^{7}$

This is the first analysis of a relatively large group at one referral centre and demonstrates the association with pulmonary tuberculosis, $50 \%$ of the cases having a history of tuberculosis, most of whom were British. Only one case was associated with histoplasmosis - in contrast to the situation in the USA where histoplasmosis is the most frequently implicated aetiological factor ${ }^{8}$ - and even this case had a history of previous pulmonary tuberculosis. Mediastinal lymphadenopathy can be a prominent feature in some cases of adult tuberculosis in the UK, especially in immigrants, and has been reported as progressing to sclerosing mediastinitis in some. ${ }^{9}$ There was no evidence of any other infective agent playing a part in the aetiology of sclerosing mediastinitis in our study. No active granulomas or infectious agent could be found in the resected tissues, which is the usual finding when fibrosis is extensive. ${ }^{4}$

Surgery was necessary in most of the cases for a specific diagnosis as suspicion of malignancy was high. It is essential to sample extensively in order not to miss an underlying neoplasm as there can be considerable fibrosis associated with some tumours, especially lymphomas. In all patients it was not possible to resect the fibrous tissue, and bypass of the superior vena cava was undertaken in six cases with good results which have been reported previously. ${ }^{10}$ However, others have found surgery for bypass difficult with poor results. ${ }^{11}$

The pathogenesis of extensive progressive fibrosis within the mediastinum remains unclear. Kunkel et $a^{12}{ }^{12}$ suggested that mediastinal fibrosis resulted from traumatic rupture of granulomatous lymph nodes. Goodwin et $a l^{13}$ proposed the currently most acceptable hypothesis that sclerosing mediastinitis results from a delayed hypersensitivity reaction to fungal, mycobacterial, or other unknown antigens. The history of tuberculosis in half of our patients, the infiltration of the fibrous mass by plasma cells and lymphocytes, and the raised serum immunoglobulin levels support an immunological reaction. The use of antifungal and antituberculous agents has been advocated in cases with evidence of active granulomas ${ }^{14}$ but these, as well as steroids, are generally not useful in established cases with extensive fibrosis. ${ }^{15}$ Most reports emphasise the benign nature of the condition and advocate minimal intervention unless symptoms become life threatening. ${ }^{15}$

The authors wish to thank Mr SC Lennox, Mr P Goldstraw, and Mr D Kaplan for access to their patients and Miss Pinuccia Mole for typing the manuscript. 
1 Oulmont N. Des oblitarations de la veine cava superieure. Paris: JB Baillère, 1855

2 Osler W. On obliteration of the superior vena cava. Bull fohns Hopkins Hosp 1903;14:169-82.

3 Knox LB. Chronic mediastinitis. Am f Med Sci 1925;169: 807-20.

4 Schowengerdt CG, Suyemoto R, Beachley F. Granulomatous and fibrous mediastinitis. A review and analysis of 180 cases. F Thorac Cardiovasc Surg 1969;57:365-79.

5 Marchevsky AM, Kaneko M. Inflammatory diseases of the mediastinum. In: Surgical pathology of the mediastinum. New York: Raven Press, 1984:16-28.

6 Graham JR, Suby HI, LeCompte PR, Sadowsky NL. Fibrotic disorders associated with methysergide therapy for rotic disorders associated with methysergice

7 Comings DE, Skubi KB, Van Eyes J, Motulski AG. Familial multifocal fibrosclerosis. Findings suggesting that retroperitoneal fibrosis, mediastinal fibrosis, sclerosing cholangitis, Riedel's thyroiditis and pseudotumour of the orbit may be different manifestations of a single disease. Ann Intern Med 1967;66:884-92.

8 Anonymous. Case records of the Massachusetts General
Hospital. Weekly clinicopathological exercises. Case 61989. A 57-year-old man with increasing dyspnea and a mediastinal mass. N Engl f Med 1989;320:380-9.

9 Bloomberg TJ, Dow CJ. Contemporary mediastinal tuberBloomberg TJ, Dow CJ. Contempor
culosis. Thorax 1980;35:392-6.

10 Mitchell IM, Saunders NR, Maher O, Lennox SC, Walker DR. Surgical treatment of idiopathic mediastinal fibrosis: report of five cases. Thorax 1986;41:210-4.

11 Strimlan CV, Dines DE, Payne WS. Mediastinal granuloma. Mayo Clin Proc 1975;50:702-5.

12 Kunkel WM Jr, Clagett OT, McDonald JR. Mediastinal granuloma. $\mathcal{F}$ Thorac Surg 1954;27:565-74.

13 Goodwin RA, Mickell JA, Des Prez RM. Mediastinal fibrosis complicating healed primary histoplasmosis and tubercomplicating healed primary hist
culosis. Medicine 1972;51:227-46.

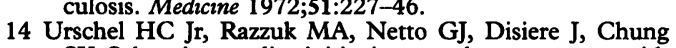
SY. Sclerosing mediastinitis: improved management with histoplasmosis titer and ketoconazole. Ann Thorac Surg 1990;50:215-21.

15 Dines DE, Payne WS, Bernatz PE, Pairolero PC. Mediastinal granuloma and fibrosing mediastinitis. Chest 1979;75:320-4. 